\title{
ABORTO BOVINO POR Neospora caninum NO RIO GRANDE DO SUL
}

\section{BOVINE ABORTION DUE TO Neospora caninum IN RIO GRANDE DO SUL STATE, BRAZIL}

\author{
Luís Gustavo Corbellini $^{1}$ David Driemeier ${ }^{2}$ Cláudio Cruz $^{1}$ Marcelo Maronna Dias $^{3}$
}

RESUMO

Casos de aborto bovino submetidos ao Setor de Patologia da Faculdade de Veterinária da Universidade Federal do Rio Grande do Sul entre os anos de 1995 e o primeiro trimestre de 1999 foram selecionados e analisados histologicamente. Um total de 30 fetos abortados foram avaliados através da coloração de Hematoxilina e Eosina (HE) e lesões inflamatórias não supurativas foram encontradas principalmente no cérebro elou coração de seis casos. Realizou-se imunoistoquímica pela técnica de streptavidina-biotina, utilizando-se anticorpos anti-Neospora caninum e antiToxoplasma gondii nesses seis casos. Taquizoítos marcados pelo anticorpo anti-N. caninum foram visualizados em três dos seis fetos bovinos testados e não houve reação com o anticorpo antiT. gondii. Esses resultados comprovam a existência de aborto bovino causado por N. caninum no Estado do Rio Grande do Sul.

Palavras-chave: Neospora caninum, aborto; bovino, imunoistoquímica.

\section{SUMMARY}

Aborted bovine fetuses submitted to the Pathology Laboratory at Universidade Federal do Rio Grande do Sul, Brazil between 1995 and the first trimester of 1999 were selected and examined histologically. Tissue from thirty aborted fetuses were examined after staining with Hematoxylin \& Eosin and nonsuppurative inflammations were observed mainly in the brain and/or heart of six cases. The avidin-biotin immunohistochemistry technique was done using antiserum against Neospora caninum and Toxoplasma gondii in those six cases. Tachyzoites that reacted with $N$. caninum antisera were seen in three of the six fetuses with non-suppurative inflammation. There was no reaction with $\boldsymbol{T}$. gondii antiserum. These results confirm the presence of
Neospora caninum abortion in cattle in the state of Rio Grande do Sul, Brazil.

Key words: Neospora caninum, Abortion, bovine,

\section{INTRODUÇÃO}

Neospora caninum é um protozoário pertencente ao filo Apicomplexa semelhante ao Toxoplasma gondii, mas apresenta diferenças estruturais e antigênicas (DUBEY et al., 1988). Foi identificado pela primeira vez por DUBEY et al. (1988) em cães com encefalomielite e, antes dessa descrição, era confundido com $\boldsymbol{T}$ gondii. Taquizoítos, cistos contendo bradizoítos e oocistos, são os estágios infectantes identificados de $\boldsymbol{N}$. caninum (DUBEY \& LINDSAY, 1996; McALLISTER et al., 1998; LINDSAY et al.,1999). Os cistos são encontrados principalmente no sistema nervoso central (SNC) e taquizoítos já foram observados em células de diversos órgãos de animais infectados (DUBEY \& LINDSAY, 1996). Com relação ao ciclo evolutivo, os cães são os hospedeiros definitivos (McALLISTER $\boldsymbol{e t}$ al., 1998; LINDSAY et al., 1999) e eliminam oocistos nas fezes, contaminando o ambiente. Bovinos, caprinos, ovinos, caninos, eqüinos e cervídeos são os hospedeiros intermediários e

${ }^{1}$ Médico Veterinário, Mestrando, Universidade Federal do Rio Grande do Sul (UFRGS), Faculdade de Veterinária, Setor de Patologia.

${ }^{2}$ Médico Veterinário, Professor Adjunto Setor de Patologia Veterinária, UFRGS. Bolsista CNPq 301076/93-6. Av Bento Gonçalves 9090, CP 15094, 91540-000, Porto Alegre RS. E-mail: davetpat@ vortex.ufrgs.br. Autor para correspondência.

${ }^{3}$ Médico Veterinário, Mestrando, UFRGS, Faculdade de Veterinária, Setor de Entomozooses. Recebido para publicação em 27.10.99. Aprovado em 01.03.00 
infectam-se através da ingestão de oocistos (BARR, 1998). Há evidências da transmissão transplacentária de $N$. caninum em bovinos de leite, sugerindo que infecções por $\boldsymbol{N}$. caninum (neosporose) podem persistir dentro de uma propriedade leiteira (ANDERSON et al., 1997). Casos de neosporose foram diagnosticados em eqüinos (MARSH et al., 1996), caprinos (BARR et al., 1992), ovinos (DUBEY \& LINDSAY, 1996), cervídeos (WOODS et al., 1994) e bezerros com encefalomielite (BARR et al., 1991).

N. caninum é um importante agente etiológico de aborto em bovinos em grande parte do mundo (THILSTED \& DUBEY, 1989; THORNTON et al., 1994; OTTER $\boldsymbol{e}$ t al., 1995; McNAMEE et al., 1996; FONDEVILA et al., 1998; MOEN et al., 1998) e é considerado a principal causa de aborto em bovinos de leite na Califórnia (ANDERSON et al., 1991). No Brasil, GONDIM \& SARTOR (1997) detectaram anticorpos contra $N$. caninum através da imunofluorescência indireta (IFI) em vacas de uma propriedade leiteira com histórico de aborto no Estado de São Paulo. Em um estudo soroepidemiológico realizado no Estado da Bahia, 14,09\% dos bovinos de leite, provenientes de 14 diferentes rebanhos, foram positivos na IFI para N. caninum (GONDIM et al., 1999).

O diagnóstico dessa enfermidade está baseado nas lesões histológicas produzidas nos tecidos dos fetos abortados (OTTER $\boldsymbol{e t}$ al., 1995), na identificação do protozoário pela técnica de imunoistoquímica (LINDSAY \& DUBEY, 1989) e em provas sorológicas utilizando-se os testes de IFI ou ELISA (DUBEY \& LINDSAY, 1996). As lesões histológicas são caracterizadas por infiltrados de células inflamatórias, compostos basicamente por mononucleares, e podem ser encontradas em todos os órgãos dos fetos abortados, principalmente SNC, coração, músculo esquelético e fígado (ANDERSON $\boldsymbol{e} \boldsymbol{t} \boldsymbol{a l}$., 1991; DUBEY \& LINDSAY, 1996). As lesões produzidas no cérebro consistem de encefalite não supurativa, caracterizada por múltiplos focos de infiltrados inflamatórios mononucleares e células da glia, geralmente ao redor de um centro de necrose (DUBEY \& LINDSAY, 1996).

Este trabalho teve como objetivo identificar casos de aborto bovino causado por $N$. caninum ocorridos em diversas localidades do Estado do Rio Grande do Sul.

\section{MATERIAL E MÉTODOS}

Casos de aborto bovino enviados ao Setor de Patologia da Faculdade de Veterinária da Universidade Federal do Rio Grande do Sul (UFRGS) entre os anos 1995 até o primeiro trimestre do ano de 1999 foram analisados através da histologia, para verificar a presença de lesões compatíveis com $\boldsymbol{N}$. caninum. Foram selecionados para análise histológica 30 casos de aborto bovino que estavam disponíveis em blocos de parafina e que continham fragmentos de cérebro, músculo cardíaco, fígado, pulmão, rim ou pelo menos um desses órgãos. Todos os tecidos haviam sido fixados em formol a $10 \%$, processados histologicamente $\mathrm{e}$ corados pela Hematoxilina e Eosina (HE).

Realizou-se imunoistoquímica empregando-se um $\mathrm{kit}^{\mathrm{a}}$ contendo anticorpo secundário conjugado com biotina e streptavidina conjugada com peroxidase (técnica de streptavidinabiotina). Utilizaram-se anticorpos primários policlonais anti-N.caninum ${ }^{b}$ com uma diluição de 1:3000 e anti-Toxoplasma gondii ${ }^{c}$ (1:2400) em todos os casos onde foram encontradas lesões inflamatórias não supurativas na análise histológica dos materiais dos 30 fetos corados com HE. A técnica de imunoistoquímica utilizada (MILLS, 1992) foi modificada, submetendo-se as lâminas histológicas a uma digestão prévia com tripsina a $0,1 \%$ por 10 minutos a $37^{\circ} \mathrm{C}$, e posterior irradiação em forno microondas de uso doméstico por dois minutos na potência máxima. Durante essa etapa, as lâminas ficavam imersas em $100 \mathrm{ml}$ de tampão citrato ( $\mathrm{pH}$ 6,0). Em cada caso testado na imunoistoquímica, havia um controle negativo no qual o anticorpo primário era substituído por soro fetal bovino.

\section{RESULTADOS}

Dos 30 fetos selecionados para análise histológica, seis apresentavam lesões histológicas compatíveis com as causadas por $N$. caninum. $\mathrm{Na}$ tabela 1, são descritas as lesões encontradas nos diversos órgãos avaliados. $O$ cérebro estava disponível para análise em quatro casos e todos apresentavam lesões inflamatórias não supurativas, multifocais, compostas por células inflamatórias mononucleares. Alguns dos focos inflamatórios distribuíam-se ao redor de um centro de necrose (Figura 1). Infiltrados inflamatórios mononucleares perivasculares 
Tabela 1 - Achados histopatológicos dos casos de aborto bovino que continham lesões compatíveis com infecção por Neospora caninum.

\begin{tabular}{|c|c|c|c|c|}
\hline Caso & Ano & $\begin{array}{l}\text { Período } \\
\text { (meses) }\end{array}$ & Órgãos examinados & Lesões encontradas \\
\hline 1 & 1995 & $\mathrm{ND}^{1}$ & $\begin{array}{l}\text { Coração, pulmão, fígado, } \\
\text { rim. }\end{array}$ & $\begin{array}{lrr}\begin{array}{l}\text { Miocardite não } \\
\text { extensos }\end{array} & \begin{array}{r}\text { inurativa com } \\
\text { infiltrados }\end{array} \\
\text { subendocárdicos e } & \text { áreas de } \\
\text { necrose com } & \text { alguns } \\
\text { polimorfonucleares. } & \end{array}$ \\
\hline 2 & 1996 & 7,5 & Cérebro. & 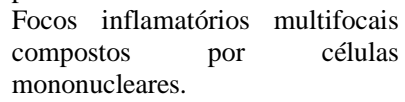 \\
\hline 3 & 1997 & ND & Cérebro. & $\begin{array}{l}\text { Focos inflamatórios } \\
\text { compostos multifocais } \\
\text { mononucleares cor com necrose } \\
\text { central em algumas áreas. }\end{array}$ \\
\hline 4 & 1998 & 6 & $\begin{array}{l}\text { Cérebro, coração, pulmão, } \\
\text { fígado, rim. }\end{array}$ & 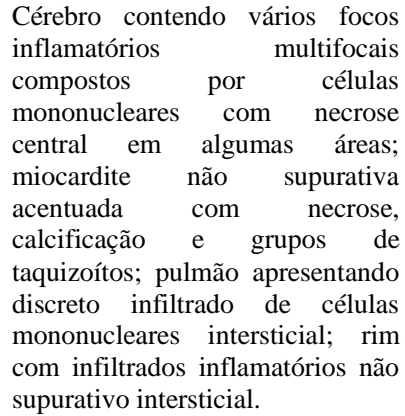 \\
\hline 5 & 1998 & ND & Coração. & $\begin{array}{l}\text { Miocardite não supurativa } \\
\text { moderada. }\end{array}$ \\
\hline 6 & 1999 & 5 & $\begin{array}{l}\text { Cérebro, coração, pulmão, } \\
\text { fígado. }\end{array}$ & $\begin{array}{l}\text { Cérebro com focos inflamatórios } \\
\text { multifocais compostos por células } \\
\text { mononucleares com necrose } \\
\text { central em algumas áreas; } \\
\text { miocardite não supurativa; pulmão } \\
\text { com focos de necrose e infiltrados } \\
\text { de células mononucleares } \\
\text { intersticial; figado com áreas de } \\
\text { necrose focal. }\end{array}$ \\
\hline
\end{tabular}

inflamatórios no cérebro (Figura 5). Cistos não foram visualizados em nenhum dos tecidos dos fetos onde realizou-se a imunoistoquímica. Em alguns cortes de cérebro, havia numerosas lesões multifocais não supurativas, mas apenas em poucas áreas encontrou-se taquizoítos no centro destas lesões. A imunoistoquímica foi positiva apenas no miocárdio do caso 4, onde, em algumas partes, havia numerosos taquizoítos (Figura 6). Não houve reação com o anticorpo anti-T. gondii em nenhum dos casos analisados.

Dentre os seis fetos que continham lesões, quatro foram provenientes de propriedades de leite, um de propriedade de corte e um não foi possível estabelecer a procedência. Os rebanhos leiteiros nos quais diagnosticou-se neosporose tinham em média 100 animais da raça holandês em ordenha e eram confinados em instalações do tipo "free-stall”. Em um dos estabelecimentos, havia histórico de aborto e foram analisados quatro fetos submetidos ao setor de patologia durante um intervalo de três anos. Dois desses foram positivos para $N$. caninum na imunoistoquímica. Em um outro rebanho leiteiro, foram enviados dois fetos em um ano, sendo que um foi positivo no teste imunoistoquímico.

\section{DISCUSSÃO} em um caso. Lesões histológicas também estavam presentes no pulmão, fígado e rim (Tabela 1).

Dos seis casos de aborto com lesões inflamatórias não supurativas, compatíveis com infecção por $\boldsymbol{N}$. caninum, três foram confirmados pela imunoistoquímica. $\mathrm{Na}$ tabela 2, encontram-se os resultados obtidos na imunoistoquímica. Através dessa técnica, observaram-se taquizoítos marcados pelo anticorpo anti-N. caninum em três dos quatro cérebros que continham lesões (Figura 3 ) e no miocárdio de um caso (Figura 4). Os taquizoítos encontravam-se em número variável no centro dos focos
O diagnóstico de aborto por $\boldsymbol{N}$. caninum baseou-se na presença de lesões histológicas e reação positiva na imunoistoquímica. Casos de neosporose bovina foram confirmados pela imunoistoquímica em três dos trinta fetos bovinos analisados (10\%). Esses valores correspondem a uma estimativa da prevalência de casos de aborto por $\boldsymbol{N}$. caninum no Rio Grande do Sul. Muitos casos antigos não foram avaliados porque não estavam disponíveis, o que poderia alterar esses resultados. A incidência de aborto bovino por $N$. caninum 


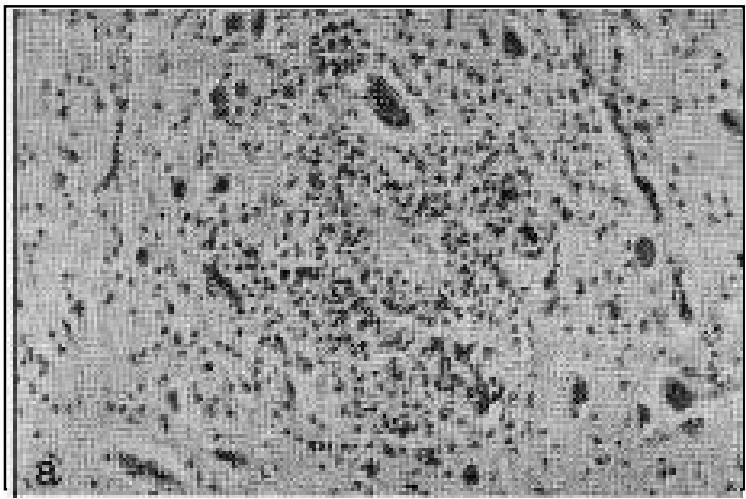

Figura 1 - Encefalite focal. Infiltrado inflamatório composto por células mononucleares ao redor de um centro de necrose. Cérebro. Feto bovino. HE, obj. 20.

diagnosticado pela imunoistoquímica em outros trabalhos varia de 4,2\% na Inglaterra e Irlanda do Norte (OTTER $\boldsymbol{e}$ t al., 1995; McNAMEE $\boldsymbol{e}$ t al., 1996) a $19 \%$ na Califórnia (ANDERSON et al., 1991).

Dos quatro casos em que havia encefalite focal, três foram positivos na imunoistoquímica (75\%). Apesar do pequeno número de casos, verificou-se uma alta correlação entre encefalite focal e infecção por $N$. caninum, como descrito por ANDERSON et al.(1991) que constatou que $83 \%$ dos casos que continham lesões características no SNC foram confirmadas pela imunoistoquímica. A imunoistoquímica realizada no coração revelou a presença de taquizoítos em apenas um de quatro casos com miocardite não supurativa. Esse resultado, provavelmente seja decorrente do pequeno número de taquizoítos presentes nos tecidos dos fetos (NIETFELD $\boldsymbol{e t}$ al., 1992). WOUDA $\boldsymbol{e} \boldsymbol{t}$ al.(1997) verificaram que $14 \%$ dos corações foram positivos na imunoistoquímica comparados com $85 \%$ de

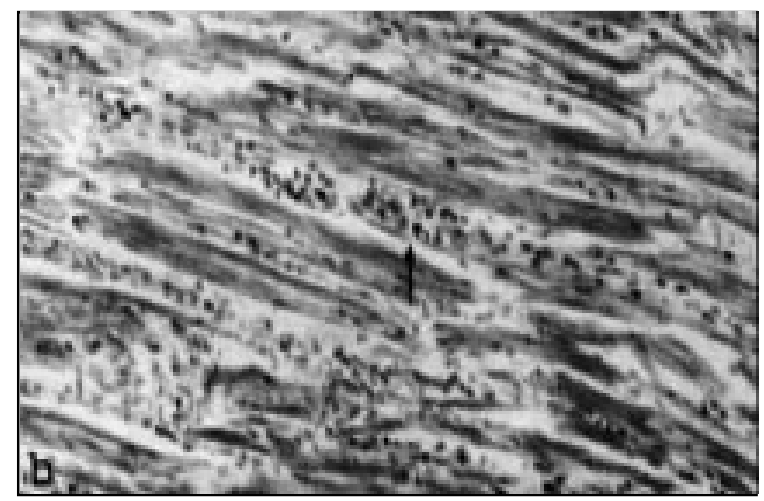

Figura 2 - Miocardite não supurativa. Infiltrado inflamatório mononuclear entre as fibras musculares cardíacas (seta). Coração. Feto bovino. HE, obj. 20.
Tabela 2 - Resultados da imunoistoquímica realizada nos casos de aborto bovino com lesões não supurativas compatíveis com Neospora caninum.

\begin{tabular}{ccc}
\hline Caso & Órgão Realizado & Resultados \\
& & \\
1 & Coração & - \\
2 & Cérebro & - \\
3 & Cérebro & + \\
4 & Cérebro & + \\
& Coração & + \\
5 & Coração & - \\
6 & Cérebro & + \\
& Coração & - \\
\end{tabular}

cérebros positivos neste teste. A visualização de taquizoítos foi facilitada pela imunoistoquímica, já que estes foram observados através da coloração de HE somente no miocárdio de um feto. A forma de cisto não foi encontrada em nenhum caso investigado neste trabalho e, conforme alguns autores (ANDERSON, et al., 1991; HELMAN, et al., 1998), os cistos são geralmente encontrados fora dos focos de lesões e em pequeno número. Lesões não supurativas também estavam presentes no pulmão, fígado e rim, indicando que a infecção por $N$. caninum é sistêmica e pode atingir vários tecidos dos fetos bovinos infectados.

Os casos de aborto deste trabalho foram avaliados apenas para $N$. caninum $e \boldsymbol{T}$. gondii, sendo importante salientar que outras causas poderiam estar implicadas ao mesmo tempo e que os achados histopatológicos poderiam ser coincidentes. OTTER $\boldsymbol{e t}$ al.(1995) encontraram evidências de infecções causadas por Leptospira hardjo, Pasteurella

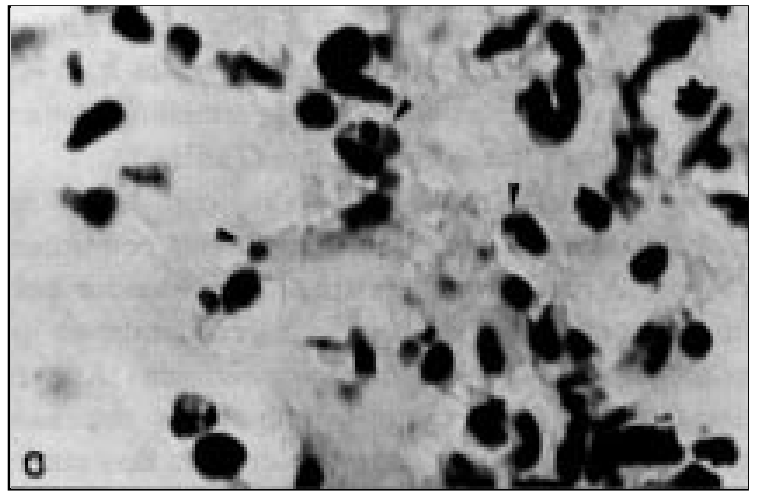

Figura 3 - Presença de taquizoítos (ponta de seta) marcados com anticorpos anti-Neospora caninum. Cérebro. Feto bovino. Imunoistoquímica. Streptavidina-biotina, obj. 100.

Ciência Rural, v. 30, n. 5, 2000. 


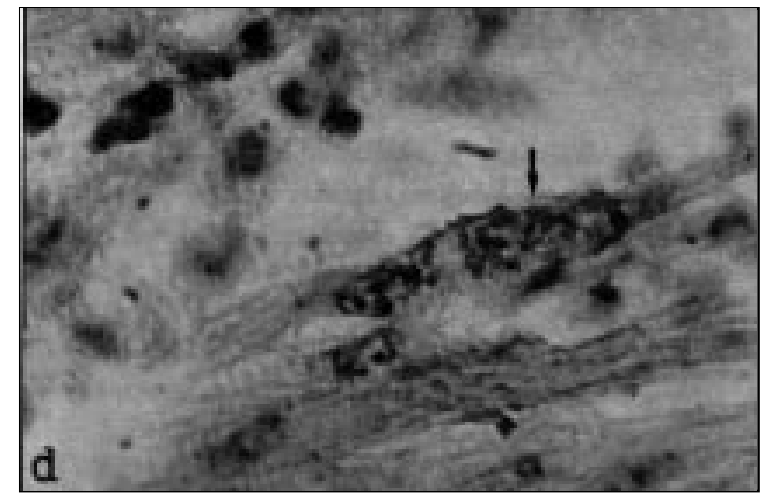

Figura 4 - Grupos de taquizoítos no citoplasma de uma célula muscular cardíaca marcados com anticorpos antiNeospora caninum (seta). Coração. Feto bovino. Imunoistoquímica. Streptavidina-biotina, obj. 100.

haemolytica e Bacillus licheniformis em casos de fetos bovinos que continham lesões inflamatórias não supurativas.

Todos os casos positivos na imunoistoquímica foram provenientes de rebanhos de leite criados em instalações do tipo "free-stall". Em uma propriedade com histórico de aborto, foram encontrados dois casos positivos. Muitos dos relatos apontam que $N$. caninum é uma importante causa de abortos em bovinos de leite (ANDERSON $\boldsymbol{e} \boldsymbol{t} \boldsymbol{a l}$., 1991; NIETFIELD et al., 1992). No presente trabalho, não foi possível determinar a real incidência de aborto por $\boldsymbol{N}$. caninum através de técnicas histológicas, pois muitos abortos não são submetidos a diagnóstico histológico.

Os resultados deste estudo indicam que $10 \%$ dos abortos avaliados estavam infectados com $N$. caninum, podendo ser responsável por consideráveis perdas econômicas. Aborto por $N$.

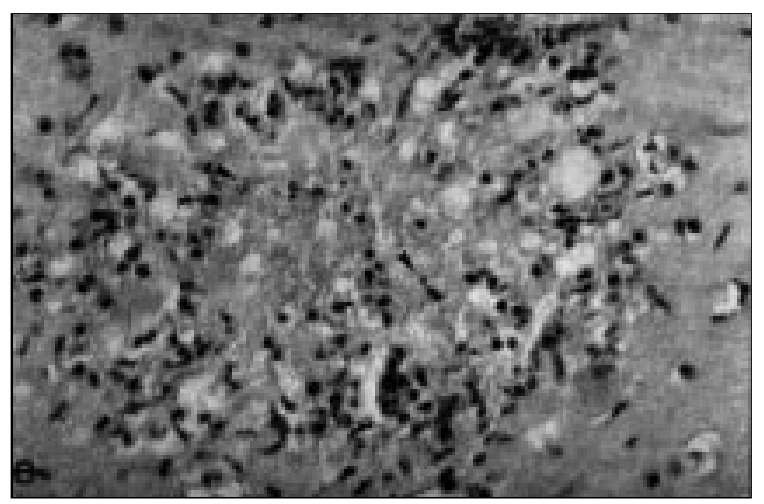

Figura 5 - Necrose focal contendo taquizoítos (ponta de seta) marcados com anticorpos anti-Neospora caninum. Cérebro. Feto bovino. Imunoistoquímica. Streptavidina-biotina, obj. 40.

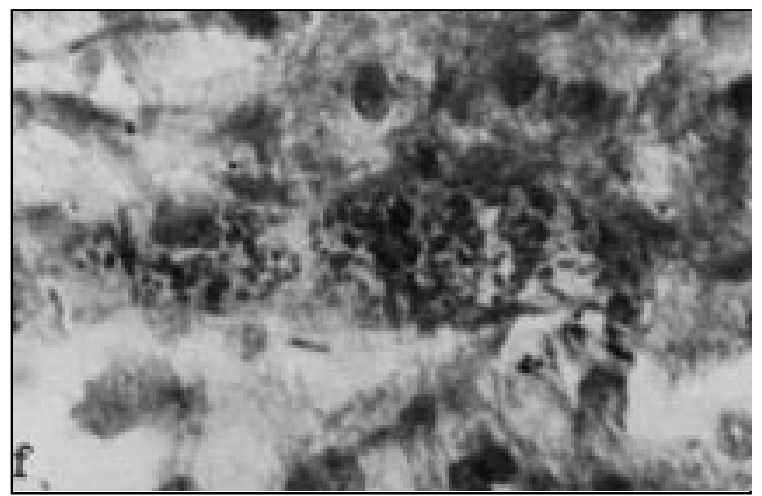

Figura 6 - Grande número de taquizoítos no miocárdio. Coração. Feto bovino. Imunoistoquímica. Streptavidina-biotina, obj. 100.

caninum está presente no Estado do Rio Grande do Sul e suas causas e fatores predisponentes devem ser melhor avaliados para que medidas preventivas possam ser tomadas.

\section{FONTES DE AQUISIÇÃO}

a Dako LSAB + Kit, Peroxidase, Carpinteria, USA

b VMRD, Pullman, USA

c VMRD, Pullman, USA

\section{REFERÊNCIAS BIBLIOGRÁFICAS}

ANDERSON, M.L., BLANCHARD, P.C., BARR, B.C., et al. Neospora-like protozoan infection as a major cause of abortion in California dairy cattle. J Am Vet Med Assoc, v.198, p.241-244, 1991.

ANDERSON, M.L., REYNOLDS, J.P., ROWE, J.D., et al. Evidence of vertical transmission of Neospora sp. infection in dairy cattle. J Am Vet Med Assoc, v.210, p.1169-1172, 1997.

BARR, B.C., CONRAD, P.A., DUBEY, J.P., et al. Neosporalike encephalomyelitis in a calf: pathology, ultrastructure, and immunoreactivity. J Vet Diagn Invest, v.3, p.39-46, 1991

BARR, B.C, ANDERSON, M.L., WOODS, L.W., et al. Neospora-like protozoal infections associated with abortion in goats. J Vet Diagn Invest, v.4, p.365-367, 1992.

BARR, B.C. Question: What is neosporosis? Veterinary Exchange, v.20, n.11(D), p.4, 1998. (Suplement to Compendium on Continuing Education for the Practicing Veterinarian, 1998).

DUBEY, J.P., CARPENTER, J.L., SPEER, C.A., et al. Newly recognized fatal protozoan disease of dogs. J Am Vet Med Assoc, v.192, p.1269-1285,1988.

DUBEY, J.P., LINDSAY, D.S. A review of Neospora caninum and neosporosis. Vet Parasitol, v.67, p.1-59, 1996. 
FONDEVILA, D., AÑOR, S., PUMEROLA, M., et al. Neospora caninum identification in an aborted bovine fetus in Spain. Vet Parasitol, v.77, p.187-189, 1998.

GONDIM, L.F.P., SARTOR, I.F. Detecção de anticorpos contra Neospora caninum em vacas leiteiras em uma propriedade com histórico de aborto. In: SEMINÁRIO BRASILEIRO DE PARASITOLOGIA VETERINÁRIA, 10, SEMINÁRIO DE PARASITOlOGia VeterináRIA DO MERCosul, 1, 1997, Itapema. Anais... Itajaí : CBPV, 1997. p.346.

GONDIM, L.F., SARTOR, I.F., HASEGAWA, M. et al. Seroprevalence of Neospora caninum in dairy cattle in Bahia, Brazil. Vet Parasitol, v.86, p.71-75, 1999.

HELMAN, R.G., STAIR, E.L., LEHENBAUER, T.W., et al. Neosporal abortion in Oklahoma cattle with emphasis on the distribution of brain lesions in aborted fetuses. J Vet Diagn Invest, v.10, p.292-295, 1998.

LINDSAY, D.S., DUBEY, J.P. Immunohistochemical diagnosis of Neospora caninum in tissue sections. Am J Vet Res, v.50, p.1981-1983, 1989.

LINDSAY, D.S., DUBEY, J.P., DUNCAN, R.B. Confirmation that $\operatorname{dog}$ is a definitive host for Neospora caninum. Vet Parasitol, v.82, p.327-333, 1999.

MARSH, A.E., BARR, B.C., MADIGAN, J., et al. Neosporosis as a cause of equine protozoal myeloencephalitis. J Am Vet Med Assoc, v.209, p.1907-1913, 1996.

MILLS, B. Immunohistochemistry. In: PROPHET, E.B., MILLS, B., ARRINGTON, J.B. et al. Laboratory methods in histotechnology. Washington: AFIP, 1992. Cap.23, p.247-255.

McALLISTER, M.M., DUBEY, J.P., LINDSAY, D.S., et al. Dogs are definitive hosts of Neospora caninum. nt $\mathbf{J}$ Parasitol, v.28, p.1473-1478, 1998.
McNAMEE, P.T., TREES, A.J., GUY, F., et al. Diagnosis and prevalence of neosporosis in cattle in Northern Ireland. Vet Rec, v.138, p.419-420, 1996.

MOEN, A.R., WOUDA, W., MUL, M.F. Increased risk of abortion following Neospora caninum abortion outbreaks: A retrospective and prospective cohort study in four dairy herds. Theriogenology, v.49, p.1301-1309, 1998.

NIETFELD, J.C., DUBEY, J.P., ANDERSON, M.L., et al. Neospora-like protozoan infection as a cause of abortion in dairy cattle. J Vet Diagn Invest, v.4, p.223-226, 1992.

OTTER, A., JEFFREY, M., GRIFFITHS, I.B., et al. A survey of the incidence of Neospora caninum infection in aborted and stillborn bovine fetuses in England and Wales. Vet Rec, v.136, p.602-606, 1995.

THILSTED, J.P., DUBEY, J.P. Neosporosis-like abortions in a herd of dairy cattle. J Vet Diagn Invest, v.1, p.205-209, 1989.

THORNTON, R.N., GAJADHAR, A., EVANS, J. Neospora abortion epidemic in a dairy herd. $\mathbf{N} \mathbf{Z}$ Vet J, v.42, p.190191, 1994.

WOODS, L.W., ANDERSON, M.L., SWIFT, P.K., et al. Sistemic neosporosis in a California black-tailed deer (Odocoileus hemionus columbianus). J Vet Diagn Invest, v.6, p.508-510, 1994.

WOUDA, W., MOEN, A.R., VISSER, I.J.R.,et al. Bovine fetal neosporosis: a comparison of epizootic and sporadic abortion cases and different age classes with regard to lesion severity and immunohistochemical identification of organisms in brain, heart, and liver. J Vet Diagn Invest, v.9, p.180-185, 1997. 